Conclusion: his study showed that survivin binds to DNA and regulates the core gene expression contributing to maturation and function of the IFNg producing Th1 cells.

References: -

Disclosure of Interests: Malin Erlandsson: None declared, Karin ME Andersson: None declared, Nisha Nair: None declared, Anastasius Damdimopoulos: None declared, Sofia Töyrä Silfverswärd: None declared, Rille Pullerits: None declared, Anne Barton Consultant of: AbbVie, Maria I Bokarewa: None declared

DOI: 10.1136/annrheumdis-2020-eular.4325

\section{OP0128 \\ DISTINCT FEATURES OF HLA-DR+ AND HLA-DR- PD-1HI CXCR5-T PERIPHERAL HELPER CELLS IN SEROPOSITIVE RHEUMATOID ARTHRITIS}

H. Yamada ${ }^{1}$, T. Sasaki ${ }^{1}$, K. Suzuki ${ }^{1}$, M. Takeshita ${ }^{1}$, S. Tanemura ${ }^{2}$, N. Seki ${ }^{2}$, H. Tsujimoto ${ }^{2}$, T. Takeuchi ${ }^{1}{ }^{1}$ Division of Rheumatology, Department of Internal Medicine, Keio University School of Medicine, Tokyo, Japan, Tokyo, Japan; ${ }^{2}$ Mitsubishi Tanabe Pharma Corporation, Kanagawa, Japan, Kanagawa, Japan

Background: PD-1hi CXCR5-T peripheral helper (Tph) cells are newly identified pathogenic CD4+ helper T cells in rheumatoid arthritis (RA). Since Tph cells have been emerged quite recently, the characteristics of Tph cells as a biomarker of RA are not fully understood.

Objectives: The aim of the study is to evaluate how useful Tph cells in peripheral bloods are when compared to other immune cell subsets, and to clarify which Tph subset most accurately reflects the disease activity of RA.

Methods: The RA patients who visited our rheumatology department between January 2000 and February 2017, and met the 2010 ACR/European League Against Rheumatism (EULAR) classification criteria were included. We first assessed correlation with 40 immune cell subsets and the disease activity of RA. Next, the proportions of these immune cells were compared between RA and healthy controls (HCs). We also investigated the immune cell subsets which reflected the time course change of the disease activity after the methotrexate (MTX) treatment. The study protocol was approved by the ethics committee at Keio University School of Medicine.

Results: Thirty-four seropositive RA, 12 seronegative RA and 34 HCs were included. The Immune cell subsets which showed correlation with DAS28-ESR $(r>0.2$ or $r>-0.2)$ were activated CD4 T cells $(r=0.31)$, HLA-DR+Th1 cells $(r=$ $0.20), \mathrm{HLA}-\mathrm{DR}+\mathrm{Th} 1-17$ cells $(r=0.25)$, Tfh $1-17$ cells $(r=-0.25), \mathrm{HLA}-\mathrm{DR}+\mathrm{Tph}$ cells $(r=0.22), C D 3+C D 8+$ naïve T cells $(r=-0.25), C D 3+C D 8+$ effector memory $T$ cells $(r=-0.26)$, plasma cells $(r=0.40)$ and CD14++CD16+intermediate monocyte $(r=$ 0.23). The proportions of HLA-DR+Th1 cells (2.3\% vs. $5.7 \%)$, HLA-DR+Th1-17 cells $(0.7 \%$ vs. $2.2 \%)$, Tfh $1-17$ cells $(1.7 \%$ vs. $2.0 \%)$, HLA-DR+Tph cells $(0.02 \%$ vs. $0.1 \%)$, CD3+CD8+effector memory T cells (16.6\% vs $25.7 \%)$, plasma cells $(0.04 \%$ vs. $0.17 \%)$ were statistically higher in the patients with RA compared to $\mathrm{HCs}$. While the proportion of Tph cells showed weak correlation with DAS28-ESR $(r=0.18)$, that was extremely higher in RA $(0.08 \%$ vs. $0.25 \%)$. Interestingly, when assessing the correlations with the disease activity in seropositive and seronegative RA separately, the proportions of Tph cells $(r=0.52)$ and HLA-DR+Tph cells $(r=0.50)$ were highly reflected in seropositive RA, but not in seronegative RA. Regarding the disease activity after the MTX treatment, the change of proportion of Tph cells between week 0 and 52 significantly reflected the change of DAS28-ESR ( $r=0.75, p=0.025)$, but not HLA-DR+Tph cells because of the non-specific reduction by the MTX treatment. Rather, HLA-DR-Tph cells significantly reflected the change of DAS28-ESR while receiving the MTX treatment $(r=0.76, p=0.021)$

Conclusion: Tph cells and HLA-DR+Tph cells highly reflected the disease activity of seropositive RA. However, after the treatment, the proportion of HLADR+Tph cells decreased independent from the disease activity, and that of HLADR-Tph cells more accurately reflected the change of the disease activity during the treatment.

References:

[1] Rao DA, et al. Pathologically expanded peripheral T helper cell subset drives B cells in rheumatoid arthritis. Nature. 2017;542:110-114.

Disclosure of Interests: Hiroki Yamada: None declared, Takanori Sasaki: None declared, Katsuya Suzuki: None declared, Masaru Takeshita: None declared, Shuhei Tanemura Employee of: I am employed by Mitsubishi Tanabe Pharma Corporation, Noriyasu Seki Employee of: I am employed by Mitsubishi Tanabe Pharma Corporation, Hideto Tsujimoto Employee of: I am employed by Mitsubishi Tanabe Pharma Corporation, Tsutomu Takeuchi Grant/research support from: Eisai Co., Ltd, Astellas Pharma Inc., AbbVie GK, Asahi Kasei Pharma Corporation, Nippon Kayaku Co., Ltd, Takeda Pharmaceutical Company Ltd, UCB Pharma, Shionogi \& Co., Ltd., Mitsubishi-Tanabe Pharma Corp., Daiichi Sankyo Co., Ltd., Chugai Pharmaceutical Co. Ltd., Consultant of: Chugai Pharmaceutical Co Ltd, Astellas Pharma Inc., Eli Lilly Japan KK, Speakers bureau: AbbVie GK,
Eisai Co., Ltd, Mitsubishi-Tanabe Pharma Corporation, Chugai Pharmaceutical Co Ltd, Bristol-Myers Squibb Company, AYUMI Pharmaceutical Corp., Eisai Co., Ltd, Daiichi Sankyo Co., Ltd., Gilead Sciences, Inc., Novartis Pharma K.K., Pfizer Japan Inc., Sanofi K.K., Dainippon Sumitomo Co., Ltd. DOI: 10.1136/annrheumdis-2020-eular.5552

\section{OP0129 AUTOREACTIVE, CITRULLINATED PEPTIDE-SPECIFIC T CELLS IN RHEUMATOID ARTHRITIS}

J. Swift $^{1}$, J. Stanway ${ }^{1}$, C. Hilkens ${ }^{1}$, A. Anderson ${ }^{1}$, A. Pratt ${ }^{1}$, J. Isaacs ${ }^{1}$.

${ }^{1}$ Newcastle University, Newcastle upon Tyne, United Kingdom

Background: Self-reactive CD4+ T cells are thought to play a key role in the pathogenesis of rheumatoid arthritis (RA) and represent a target for antigen-specific, tolerogenic therapies ${ }^{1}$. Phenotyping such cells could provide the means with which to monitor these treatments. Here, we aimed to demonstrate antigen-induced $T$ cell responses to RA relevant epitopes using peptide stimulation of peripheral blood mononuclear cells (PBMC). Several autoantigens have been described in RA, including various citrullinated peptide (cit-peptide) epitopes ${ }^{2}$; here we have used a combination of cit-peptides that will be used to load tolerogenic dendritic cells in a forthcoming experimental medicine study (AuToDeCRA II).

Objectives: To detect peripheral blood $\mathrm{T}$ cell proliferation in response to candidate autoantigens in RA patients and healthy controls (HCs) using flow cytometry.

Methods: PBMC from RA patients and HCs were obtained following informed consent and were cryopreserved. Prior to use, cells were thawed in medium supplemented with human serum and were labelled with a proliferation tracking dye (PTD). $2 \times 10^{5}$ labelled cells were plated into 96 well plates with a minimum of 7 replicates per condition and were either cultured alone or stimulated with individual peptides at varying concentrations or a peptide pool. On day 9 , cells from replicate wells were harvested, pooled and stained for surface markers and viability. Samples were acquired on a BD Fortessa X20 and analysis was performed in FlowJo version 10. A manual gating strategy was used to identify CD45RO+ (memory) CD4+ T cells and amongst this population, activated/proliferated cells were defined as CD25+ PTD low. Stimulation indices (SI) were calculated as the percentage of activated/proliferated cells from stimulated wells divided by the percentage from unstimulated wells.

Results: Ten RA patients (including early and established disease) were recruited alongside seven $\mathrm{HCs}$. Cit-peptides were initially titrated on an individua basis to determine the optimum concentration for use $(n=2)$. We then compared stimulation with either single peptides or a pool $(n=2)$, and found that PBMC stimulated with the pooled cit-peptides had a higher proliferative response. Both single and pooled cit-peptide induced $\mathrm{T}$ cell proliferation was observed in both RA and HC samples (Figure 1); the largest responses were seen amongst RA

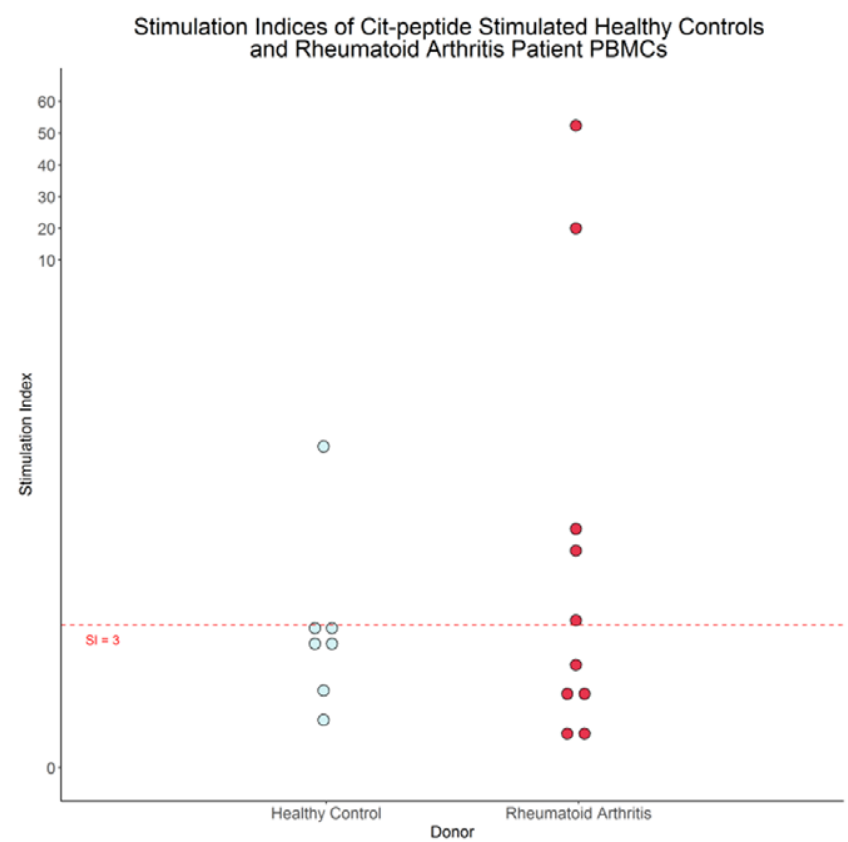

Figure 1 Antigen-specific $\mathrm{CD} 4+\mathrm{CD} 45 \mathrm{RO}+$ responses of citrullinated peptide stimulated PBMC from $\mathrm{HCs}$ and $\mathrm{RA}$ patients. 